\title{
A cross-sectorial synergies identification methodology for industrial symbiosis
}

\author{
Ogé Stéphane ${ }^{1,2,3[0000-0001-8356-6664]}$, Quintana Jean-Baptiste ${ }^{1[0000-0001-8984-5867]}$, Sockeel \\ Charles-Xavier ${ }^{10000-0002-0365-3408]}$ and, Leprince Maillère Gwenaël ${ }^{1}$, Mouhajir Mouad ${ }^{1}$, \\ Bredimas Alexandre ${ }^{1[0000-0003-1470-9283]}$ \\ ${ }^{1}$ Strane Innovation, 2 route de la noue, 91190 Gif sur Yvette, France \\ ${ }^{2}$ University of Lyon 3, CRGA-EVS UMR 5600, 18 rue Chevreul, 69632 Lyon, France \\ ${ }^{3}$ ITECH (Institut textile et chimique de Lyon), 87 Chemin des Mouilles, 69134 Ecully, France \\ stephane.ogedstrane-innovation.com
}

\begin{abstract}
Circular Economy is the model the European Union opted for to make its production and consumption system more sustainable. Industrial symbiosis is one of its operational implementation strategies. This concept aims at redesigning industries supply chains by creating new interconnections between traditionally independent chains, new sources of raw materials and new market opportunities for wasted resources. This paper introduces an innovative methodology, developed within the SCALER project that aims at facilitating substitution synergies identification between cross-sectorial supply chains. Synergy ideas are automatically generated thanks to dedicated algorithms performing matching queries on input and output data of 17 industrial sectors. Data is generic and collected from publicly available sources. The methodology's deductive approach has the benefit of proposing relevant synergy ideas for industries without asking confidential operating data. 1000 relevant synergies were already identified. Development perspectives are to reinforce the methodology with additional technical datasets such as treatment technologies, geolocated facilities databases, European economic activity/waste codes.
\end{abstract}

Keywords: Industrial Symbiosis, Input-Output matchmaking, Methodology

\section{Introduction}

The European Union (EU) is highly engaged in setting up a sustainable development. The circular economy (CE) model is seen by both politicians [1] and most academic researchers [2] as one of the solutions to achieve this goal. A CE is an economic system of stakeholders (citizens, companies, nations, etc.) who implement business models based on reducing use, reusing, recycling and/or recovering materials in production, distribution and consumption processes [3]. End-of-life and linear economy paradigms are switched to a more circular one [4] promoting a development mode that protects the environment while facilitating sustainable economic development $[5,6]$. 
Industrial symbiosis (IS) is considered as an operational strategy for CE implementation [7]. It identifies business opportunities that leverage underutilised resources, between organisations which are traditionally separate [8,9]. IS redesigns industries' supply chains and creates new interconnections (synergies) between sectors, provides new sources of secondary raw materials such as heat, combustible or material, and ensure viable market opportunities through substitution - i.e. wastes, by-products, emissions - or mutualisation - i.e. expertise, service, capacity, assets, technologies [10,11]. It improves material use rate, increases competitiveness and environmental performance of the whole industrial system and individual companies [12].

The EU invested more than $€ 130$ million since 2006 in research projects to facilitate IS uptake and dissemination thanks to methodologies, tools or networks development [13]. SCALER (SCALing European Resources with industrial symbiosis) is one of them and provides answers to targeted IS implementation issues, i.e. lack of inter-sectorial knowledge sharing, difficulties to identify and manage relevant industrial data for IS and lack of innovative synergies identification for process industries. SCALER's overall goal is to develop action plans and provide adapted solutions to process industries for a wide IS uptake. To draft tailored strategies, the EU IS potential must be assessed and its techno-economic, environmental, and social impacts must be quantified. For this purpose, a methodology to identify synergies has been created. This paper introduces the methodology, its preliminary results and some development perspectives.

\section{Background}

IS development is a journey of five steps: Opportunity identification; Opportunity assessment; Barrier removal; Commercialisation and adaptive management; Documentation, review and publication [14]. Each of them requires levers to be triggered, supported and perpetuated. Potential levers are mostly qualitative methodologies, research projects, IT tools, workshops, platforms and public programs. Focusing on IT tools, literature review reveals that most of them support the synergy identification stage, but they are not widely operational due to some limitations: geographic and sector/resource scope restriction; short lifetime due to unviable exploitation business model; lack of end-users' and industries' feedback during tools development; lack of tacit knowledge integration; inefficient ontologies [14-16]. While newer European IT tools are eliminating some of the previously detected gaps, better mechanisms are needed to make available R\&I projects progress to industries [13]. Most tools are still generally not comprehensively documented, not accessible for external participants or require additional development to be fully operational [17]. By building on past projects outcomes, SCALER provides a methodology to fulfil the IS implementation gap and thus increase the porosity between IS research and industry spheres.

The proposed methodology assists practitioners in their synergy identification journey by limiting their efforts and involvement. Characterised by its deductive approach [18], it proposes theoretical synergies based on generic information analyses. 
Methodology is mainly inspired from Looplocal [19], a tool that aims at gathering and linking varied source of information (resource composition, facility databases, LCI databases, IS experiences) in order to map the resources available in a region and propose synergy opportunities, and ISDATA [20], a web platform providing a set of information sources and a diagram with their interrelations. Some limitations are identified such as the difficulty to provide accurate synergy ideas without industrial data, and the lack of details on information sources preventing synergies identification for complex materials, with a wide variety of potential names, or requiring intermediary treatment (e.g. purification). Confidentiality issues and management of synergies for complex resources are two key challenges for a tool dedicated to process industries.

SCALER's methodology tackles these issues in building on other projects' outcomes and especially from the concept of sectorial blueprints developed in the EPOS project for 4 sectors [21]. As process industries are relatively standardised in terms of raw materials, products and emissions, generic profiles can be defined providing average qualitative and quantitative information on resources. This methodology created generic profiles for 17 sectors. Outcomes from the project eSymbiosis [22] dedicated to provide ontological solutions were also an inspiration source. Ontology is a way to integrate tacit knowledge in the methodology that not only increases the accuracy of identified direct synergies but extends the opportunities to indirect synergies thanks to additional technical knowledge such as potential uses and chemical composition. A proper ontology has been developed to match with process industry sectors' requirements.

\section{Proposed methodology}

\subsection{Scope definition}

SCALER targets in priority SPIRE's ${ }^{1}$ sectors due to their importance for the European economy and their significant environmental footprint. Solutions must be found to reinforce their competitiveness and optimise the resources use. Other sectors are added to widen the synergy opportunities range as each additional sector multiplies the chance to identify synergies. Selection process criteria are prioritised as: (1) publicly available detailed data, (2) high footprint and/or energy/material intensives, and (3) significant number of facilities in Europe. Covered sectors are shown in Table 1.

Table 1. Industrial sectors covered

\begin{tabular}{|l|l|}
\hline $\begin{array}{l}\text { SPIRE sec- } \\
\text { tors }\end{array}$ & $\begin{array}{l}\text { Cement; Ceramics; Chemicals (organic and inorganic); Non-Ferrous metals; Minerals } \\
\text { (lime); Steel; Water }{ }^{2} \text {; Engineering }\end{array}$ \\
\hline $\begin{array}{l}\text { Additional } \\
\text { sectors }\end{array}$ & $\begin{array}{l}\text { Energy (oil and gas refining, combustion plant); Glass; Fertilisers; Paper, pulp and wood; } \\
\text { Waste management (waste treatment and incineration); Food and drink; Pharmaceuticals } \\
\text { Textile }\end{array}$ \\
\hline
\end{tabular}

See: https://www.spire2030.eu/

2 These sectors are not yet integrated in the database and require manual treatment 
Technical information for these sectors is gathered in a repository. While it does not include contextual tacit knowledge (e.g. infrastructures, regulation), it integrates precious technical knowledge at several levels: Sector/Sub-sector/Process (productive systems characterisation); Resources (name, qualitative and quantitative characteristics of the resources); Elements (characteristics of the elements constituting resources).

Significant information is available in public technical sources, for free or with payable access. The main methodology sources to fill the database and test methodology results are the so-called BREFs reports (Best available techniques REFerence documents) for the sectors of interest. Complementary sources are: industrial partners; professional technical documents such as the journal Technique de l'ingénieur; academic literature (e.g. Industrial \& Engineering Chemistry Research; Journal of Cleaner Production; Journal of Hazardous Materials); and IS repositories (e.g. ISDATA [20], MAESTRI's Library of case studies[23], SCALER's Best Practices Deliverable [24]).

\subsection{Database creation}

The first methodology challenges are to analyse information sources and massively extract, organise and store quantitative data on processes and flows characteristics in a database. Several tables are created for this purpose and organised as in Fig. 1.

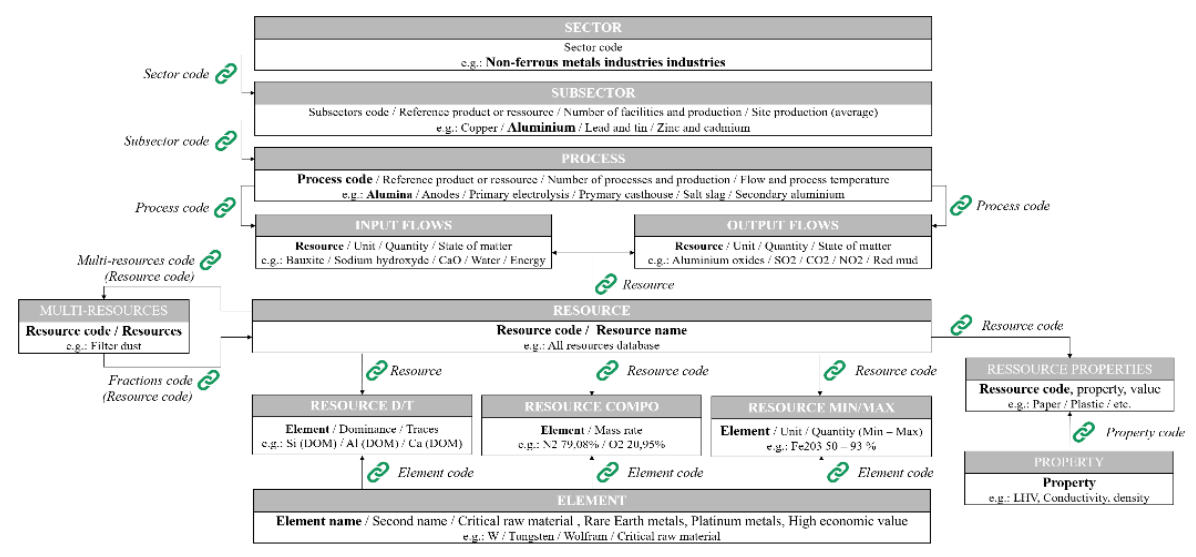

Fig. 1. Database structure and links

The table "sector" gathers the 17 covered industrial sectors. A sector is defined as a segment of an economy gathering companies with the same main productive activity (e.g. iron and steel production sector). While they are quite well standardised, production specificities exist within the same sector. Depending on their complexity, two specification levels are defined independently for each individual sector. The first level corresponds to the table "sub-sector" and is linked to "sector" through the ID sector code, defined for database's operation. A single sector might produce various type of endproducts (e.g. soda ash, phosphates, calcium carbide, etc. in the organic chemical sector), have several intermediary products (e.g. coke, sinter, etc. in the steel sector), or 
several production routes for the same standardised final product (e.g. steel production through blast furnace or electric arc furnace), influencing the used inputs and emitted outputs. "Sub-sectors" gathers information about facilities (e.g. number in Europe, volume of reference product, etc.). The second level corresponds to the table "process" and is linked to "sub-sector" through the ID subsector code (also proper to the database). Similar information as previously are gathered but at process level, i.e. different type of technologies and techniques used in a sub-sector (e.g. for the sub-sector "titanium dioxide", two processes are available: "chloride process" and "sulphate process").

All materials and energy vectors that pass through a process are considered as productive system's inputs and outputs (cf. Fig. 2). Relevant information (e.g. unit, flow rate per ton of process' reference product, state of mater) feeds respectively the tables "inputs" and "outputs" which consist in repositories detailing qualitative and quantitative characteristics of all the resources involved in industrial sectors studied.

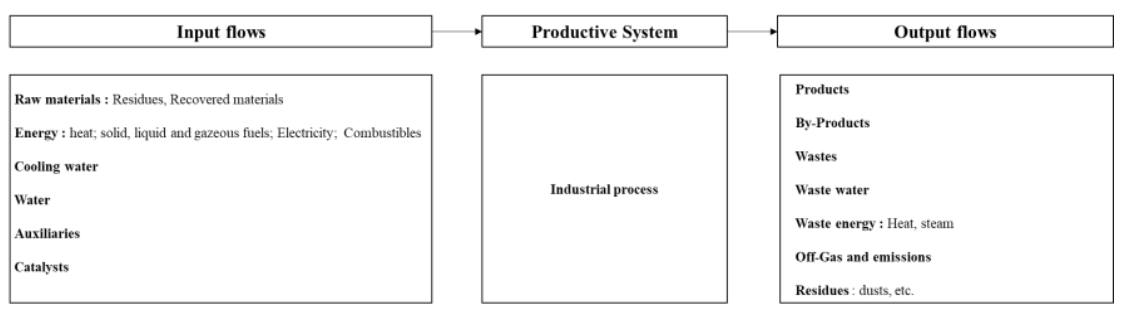

Fig. 2. Industrial process system approach

These 2 tables are linked by the ID name to the table "resource". However, the database development revealed ontological issues when defining resources' name. Many identified resources are a mix of different substances (e.g. the "Electrolyte Bleed" effluent is composed of acid and nickel fractions), some resource names are not detailed enough (e.g. the resource "salt" can refer to potassium chloride et/or sodium chloride), or resource names from the literature refer to group of resources (e.g. "Carbon Waste" in the cement sector gathers tar and bitumen). In the perspective of identifying numerous and innovative synergies, resource specification details were integrated. "resources" is thus a buffer between "input" and "output" tables and 6 additional tables for detail.

"Elements" is a table gathering all the individual chemical elements contained in "resources". This table is highly relevant in the IS context as resources might be valorized for some contents, not for the whole substance (e.g. slags are of cement industry's interest due to their silica, aluminum, calcium and iron content). "element" includes also specific characteristics, such as critical raw material status $\left[{ }^{25}\right]$, rare earth and metals, and high economic value elements. "Resource min/max", "resource composition", and "resource_dominance" are three tables providing information on the resource composition, depending on the information level gathered in the literature. They are respectively used and filled if range values (e.g. 200-500 gCH$/ 4 / \mathrm{m} 3$ of off gas), detailed compositions (e.g. $23 \% \mathrm{CO}, 45 \% \mathrm{H}_{2}, 32 \% \mathrm{CO}_{2}$ ) or qualitative indications (e.g. traces 
of sulphur) are collected. They make the link between "resource" and "element". "Multi-resources" is a table that gives the decomposition of mixed or grouped resources in individual resources (c.f. electrolyte bleed and carbon waste examples). Finally, "properties" is a table providing flexibility when attributing characteristics to each input and output. It gathers specific parameters such as: $\mathrm{pH}$, conductivity, calorific value, density, C-N-P ratio, etc. This list can be extended all along the database life.

\subsection{Synergy identification process}

Tables provide necessary information for a robust and accurate synergy identification process. The main approach is based on matching names and/or chemical element compositions of input and outputs across sectors. Depending on the synergy typologies, several matching algorithms were developed to proceed queries.

"Resource matching" is a query enabling the identification of substitution synergies when an output resource's name from a sector A corresponds to an input resource's name of a sector B (e.g. the output "Sulphuric Acid" from the steel sector corresponds to the "Sulphuric Acid" bought on the market by chemical industries). "Multi-resource matching" follows the same principle. This algorithm identifies synergies by using output and input resource's names belonging to "multi-resources" (e.g. "Fe" from the output "steel scrap" can be used to substitute a "Fe" source). "Element matching" provides more details than "Resource matching" and enables the identification of synergies through decomposition of resources into elements. Targeted queries can pinpoint recovery opportunities for high valuable elements (critical raw material, rare metals, element with a high economic value). "Heat recovery" indicates waste heat/steam recovery opportunities from process outputs to be used directly as heat or to produce steam for electricity production. "Waste as combustible" identifies all output flows which can be used for fuel preparation in waste treatment industries. "Use of alternative combustible" provides all sectors likely to use secondary fuel from waste treatments industries. "Combustible match" identifies direct combustible synergies between sectors by comparing state of matter and LHV of conventional combustibles to all other potential wastes combustibles with similar properties. Thanks to these 7 algorithms, a wide range of synergy typologies valorising varied resource natures can be identified.

\subsection{Overview of the methodology uses and users}

While this methodology has been created for a research purpose within SCALER's framework, wider uses are foreseen for several stakeholders (e.g. industries, academics, clusters, local public authorities, etc.).

The first purpose is territorial knowledge creation. The methodology can be used to make global research on all matching opportunities between sectors on a defined geographic scope. Expected results are a full list of available cross-sectorial synergies describing the territorial IS potential and the characterisation of diffuse valuable material deposits (e.g. biomass, critical raw materials). Such use can be managed by researchers 
to make accurate IS development recommendations (original SCALER purpose), or by public (e.g. local public authority) and private (e.g. facilitator) actors in different European territories and at different scales to define long-term CE strategies, make territorial marketing (e.g. foster key additional industrial activity implementation) or even give waste valorisation targets between structuring sectors.

A second goal is to facilitate R\&D. Some synergies valorising a specific resource are not implemented due to technical difficulties or lack of required treatment technologies. Public and private research centres could use the methodology to target high potential resources and develop unlocking technical solutions for IS development.

The third main use is operational and inspired from MAESTRI [26]. Users can focus on a targeted sector and identify all the potential material/energy exchanges as a receiver/emitter. For industrial companies or industry associations, it provides generic valorisation ideas for waste management and sustainable supply opportunities. For IS facilitators, they can provide their clients with new synergy ideas. Users can also focus on an individual resource/element and research valorisation opportunities. Industries, associations and facilitators might find innovative solutions for problematic streams (e.g. waste sent to landfill) and sustainable supply solutions for their raw materials (from wastes but also from traditional products if relevant). Waste management companies are also potential users in the perspective of characterising some resource deposits on territories and evaluate the possibility to build a treatment facility, massifying large amount of resources necessary to unlock business viability. For example, if a company requires hydrogen as a raw material, the "Resource matching" query identifies all hydrogen releases from other sectors process outputs. Table 2 shows that inorganic and organic chemical sectors are potential providers.

Table 2. Hydrogen synergies opportunities

\begin{tabular}{|c|c|c|}
\hline Type of data & Potential sender $\mathrm{n}^{\circ} 1$ & Potential sender $n^{\circ} 2$ \\
\hline SENDER SECTOR & INORGANIC_CHEMICALS & ORGANIC_CHEMICALS \\
\hline SENDER SUBSECTOR & SODIUM_CHLORATE & LOWER_OLEFINS \\
\hline SENDER PROCESS & SODIUM_CHLORATE_PRODUCTION & STEAM_CRACKING \\
\hline NUMBER OF FACILITIES (WHOLE SENDER SECTOR) & 15 & 39 \\
\hline BY-PRODUCT VOLUME & $6183-19236 \mathrm{t} / \mathrm{y}$ & $7000000000-100000000000 \mathrm{t} / \mathrm{y}$ \\
\hline RECEIVER SECTOR & REFINING_MINERAL_OIL_AND GAS & REFINING_MINERAL_OIL_AND GAS \\
\hline RECEIVER SUBSECTOR & HYDROCKRACKING & HYDRODESULPHURISATION \\
\hline RECEIVER PROCESS & HYDROCRACKING PROCESS & HYDRODESULPHURISATION_PROCESS \\
\hline NUMBER OF FACILITIES (WHOLE RECEIVER SECTOR) & 38 & 309 \\
\hline RECEIVER SECTOR DEMAND & $260-400 \mathrm{t}$ of h2/t of feed & 7 and 100 billion $t / y$ \\
\hline TYPE OF SYNERGY & INDIRECT & INDIRECT \\
\hline
\end{tabular}

\section{$4 \quad$ Preliminary results}

The methodology revealed to be successful as about 10000 potential synergies were identified with the different matching algorithms for the 17 sectors. They were shortlisted to the 100 most promising synergies for the purpose of SCALER. Selection criteria are partly inspired from practitioners' feedbacks [24] and are among others: variety of state of matter, synergy typology (heat, combustible, material), resource type; Homogeneous sector distribution; Economic (high market values); Strategy (rare earths and critical raw materials); High volume; High facilities number; Significant footprint. 


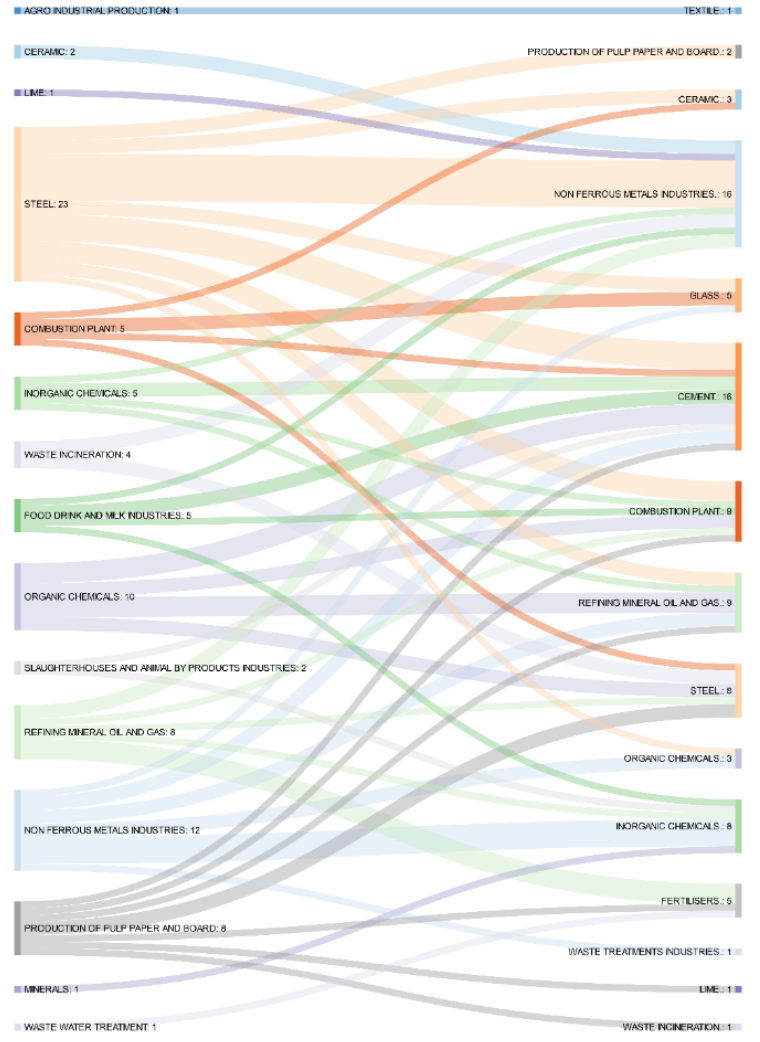

Fig. 3: Sectorial couples for the synergy sample
The sample of 100 synergies is introduced in Fig. 3, showing the sectors couples in a Sankey diagram. Steel sector has a high potential to send outputs while non-ferrous metals and cement sectors can be provided with a wide range of secondary resources. $54 \%$ of synergies valorise solid resources, a convenient state of matter for recovery and transport, while $21 \%$ are liquid and others concern gas, particles and energy. Only 21 synergies fuel-based were selected to focus further analyses on more ambitious synergies. 7 resources are listed for thermal energy recovery on nearby facilities or electricity production. 47 are direct and 52 indirect (requiring particles extraction, separation, cleaning or transformation). 1 synergy is both, depending on resource's purity.

BREFs and MAESTRI's Library of case studies [23] were used to test the methodology robustness. Among the 100 synergies, $61 \%$ are at least in one information repository. This test proves that the methodology is able to identify relevant synergies. It also shows the methodology's added value as $39 \%$ of the synergies are new and original.

\section{Conclusions and development perspectives}

An increasing number of tools supporting IS are on the market or emerging. While the majority focuses on the opportunity identification stage, none of them are dedicated to process industry for such a wide range of sectors and resources. The proposed methodology efficiently responds to IS implementation barriers and especially confidentiality and technical detail integration issues. Thanks to its deductive approach and the use of generic detailed data, the methodology generates promising synergy ideas without requiring in-site data. Results can trigger decision-makers' interest and push for further research using then industrial operating data. The methodology has a real potential to accelerate the IS dissemination by reducing efforts to identify opportunities and could 
be of interest for a wide range of stakeholders: industrial sites, industry associations, academics, IS facilitators, public authorities at different scales, and technology designers. It is an appropriate lever to implement CE strategies in industries and help redesign and complexify supply chains with new interconnections. Supply chains are more sustainable, more competitive but also more resilient by extending the supply alternatives.

Some methodology limits are identified leading to development perspectives. First, the methodology requires an important manual post-treatment to remove irrelevant synergies. Matching thresholds, depending on resources similarity rate, should be developed in queries to automatically filter results. Second, the actual volume of metadata to qualify resources is limited. Other sources of information (e.g. LCI, NACE, EWC, chemical thesaurus) are required to reinforce the existing semantic thesaurus and should lead to the creation of additional tables, associated to the existing database through hand-made correspondence tables or artificial intelligence (e.g. native language processing, web scraping). Integration of in-site data is also a perspective if the methodology is operated in a commercial way. Third, the methodology is limited to a few industrial sectors of the whole productive system. Additional sectors could be added, while for some of them it is challenging (e.g. plastic sector) as their processes are poorly standardised. Fourth, a "technology" table for existing or under development operations such as resource treatment, extraction, separation or cleaning is under development to extend the identified synergies scope and validate their technical feasibility. Finally, the development and use of the methodology currently requires a certain level of expertise and knowledge about the existing database formalism. A user-friendly interface and data integration modules should be developed for a better user experience.

\section{Acknowledgement}

This work was supported by the European Union's Horizon 2020 research and innovation program through the SCALER project (grant agreement No. 768748).

\section{References}

1. Commission, E. Closing the loop - An EU action plan for the Circular Economy. (2015).

2. Prieto-Sandoval, V., Jaca, C. \& Ormazabal, M. Towards a consensus on the circular economy. J. Clean. Prod. 179, 605-615 (2018).

3. Kirchherr, J., Reike, D. \& Hekkert, M. Conceptualizing the circular economy: An analysis of 114 definitions. Resour. Conserv. Recycl. 127, 221-232 (2017).

4. Gardetti, M. A. Introduction and the concept of circular economy. Circ. Econ. Text. Appar. 1-11 (2019). doi:10.1016/B978-0-08-102630-4.00001-7

5. Park, J., Sarkis, J. \& Wu, Z. Creating integrated business and environmental value within the context of China's circular economy and ecological modernization. J. Clean. Prod. 18, 1494-1501 (2010).

6. Ma, S., Wen, Z., Chen, J. \& Wen, Z. Mode of circular economy in China's iron and steel industry: a case study in Wu'an city. J. Clean. Prod. 64, 505-512 (2014). 
7. EIT RawMaterials. Circulator - The circular business models mixer. (2017). Available at: http://www.circulator.eu/.

8. Lombardi, D. R. \& Laybourn, P. Redefining Industrial Symbiosis. J. Ind. Ecol. 16, 2837 (2012).

9. Chertow, M. R. Industrial symbiosis : Literature and Taxonomy. Annu. Rev. Energy Environ. 25, 313-337 (2000).

10. Domenech, T., Bleischwitz, R., Doranova, A., Panayotopoulos, D. \& Roman, L. Mapping Industrial Symbiosis Development in Europe_typologies of networks, characteristics, performance and contribution to the Circular Economy. Resour. Conserv. Recycl. 141, 76-98 (2019).

11. Adoue, C. \& Chambers, S. Implementing industrial ecology: methodological tools and reflections for constructing a sustainable development. (Science Publishers, 2011).

12. Yuan, Z. \& Shi, L. Improving enterprise competitive advantage with industrial symbiosis: case study of a smeltery in China. J. Clean. Prod. 17, 1295-1302 (2009).

13. Maqbool, A. S., Mendez Alva, F. \& Van Eetvelde, G. An assessment of European information technology tools to support industrial symbiosis. Sustainability 11, 15 (2019).

14. Grant, G. B., Seager, T. P., Massard, G. \& Nies, L. Information and communication technology for industrial symbiosis. J. Ind. Ecol. 14, 740-753 (2010).

15. Fyfe, J., Mason, L., Boyle, T. \& Giurco, D. Wastenot: the streamline resource exchange background, development and case studies. (2010).

16. Holgado, M., Evans, S. \& Benedetti, M. Toolkit for Industrial Symbiosis (D4.3). (2017).

17. Benedict, M. \& Kosmol, L. Designing Industrial Symbiosis Platforms -from Platform Ecosystems to Industrial Ecosystems. in Twenty-second Pacific Asia Conference on Information Systems (2018).

18. Harpet, C. \& Gully, E. Écologie industrielle et territoriale: quels outils d'aide à la décision? De l'analyse des flux à l'approche intégrée. Déchets - Sci. Tech. 12 (2013).

19. Aid, G., Brandt, N., Lysenkova, M. \& Smedberg, N. Looplocal - a heuristic visualization tool to support the strategic facilitation of industrial symbiosis. J. Clean. Prod. 98, 328-335 (2015).

20. ISDATA. ISDATA. (2013). Available at: http://isdata.org.

21. EPOS. The EPOS sector blueprints. 1 (2017). Available at: https://www.spire2030.eu/sites/default/files/users/user222/Eposdocs/HullPC/Blueprints.pdf.

22. Cecelja, F. et al. E-symbiosis: technology-enabled support for industrial symbiosis targeting small and medium enterprises and innovation. J. Clean. Prod. 98, 336-352 (2015).

23. Evans, S., Benedetti, M. \& Holgado Granados, M. Library of Industrial Symbiosis case studies and linked exchanges. (2017). doi:https://doi.org/10.17863/CAM.12608

24. Vladimirova, D., Miller, K. \& Evans, S. Lessons learnt and best practices for enhancing industrial symbiosis in the process industry. (2018).

25. European Commission. Report on critical raw materials and the circular economy Publications Office of the EU. (2018).

26. Benedetti, M., Holgado, M. \& Evans, S. Prototype library of case studies linked to a waste database (D4.2). (2017). 